\title{
On the Lagrange libration points of the perturbed Earth-Moon System
}

\author{
Tatiana V. Salnikova ${ }^{1}$ and Sergey Ya. Stepanov ${ }^{2}$ \\ ${ }^{1}$ Moscow State Lomonosov University, Moscow, Russia \\ email: tatiana.salnikova@gmail.com \\ ${ }^{2}$ Dorodnitsyn Computing Center, RAS, Moscow, Russia \\ email: stepsj@ya.ru
}

\begin{abstract}
In this work we discuss the elusive Kordylewski clouds - dust matter in the neighborhood of the Lagrange libration points $L_{4}, L_{5}$ of the Earth-Moon system. On the base of restricted planar circular four body problem we get some proof for possibility of existence of four such clouds and some rule to predict the optimal moments of time for their observation.
\end{abstract}

Keywords. Earth, Moon, libration points, stable periodic solution, interplanetary dust clouds

\section{Introduction}

In 1961 polish astronomer K. Kordylewski took photos of the interplanetary dustclouds in the neighborhood of triangular libration point $L_{5}$. Later there were many attempts to repeat this observation or to give some theoretical explanation for this phenomenon, but almost all of them were unsuccessful.

\section{Overview}

We consider the problem of four bodies - the Earth (E), the Moon (M), the Sun (S) and a test particle $(\mathrm{P})$. As an absolute coordinate system we take the frame with origin $S$ and axes pointed to stars. Barycenter $O$ of the Earth-Moon system moves around $S$ along circular orbit with the angular velocity $\omega$, Earth and Moon rotate as a rigid body around $O$ in the plane of orbit of $O$. Let us consider the motion of the particle $\mathrm{P}$ in the coordinate system $O x y z$, rotating around the $z$-axis, which is orthogonal to the plane $(S E M)$, with absolute angular velocity $\omega_{1}, x$-axis directed along $O M$. Let $t$ be the time, $t_{0}$ - the full moon time, $p=2 \pi\left(\omega_{1}-\omega\right)\left(t-t_{0}\right)$ - the angle between radius-vector $S O$ and the $x$-axis, $T=2 \pi /\left(\omega_{1}-\omega\right)$ - the length of synodic month; $m_{0},(1-\mu) m, \mu m$ - masses of the Sun, the Earth and the Moon. And let $R=|S O|, l=|E M|, r=|P E|$. We choose $m, l, \omega_{1}^{-1}$ as units of mass, length and time. Then due to relations $\omega^{2} R^{3}=\gamma m_{0}$ and $\omega_{1}^{2} l^{3}=\gamma m$ we obtain $m=1, l=1, \omega_{1}=1, \gamma=1, m_{0}=\omega^{2} R^{3}$. For other parameters we take the values $R=389.18, \omega=1 / 13.36, \mu=0.0122$.

The Lagrange equations for the radius-vector of particle $\mathrm{P} Z(p)=(x(p), y(p), z(p))^{T}$ possess a stable in Lyapunov sense $2 \pi$-periodic solution (the black curve at top-left figure) $Z^{\star}(p)$, surrounding the libration point $L_{4}$ with initial conditions

$$
Z^{\star}\left(t_{0}\right)=(-0.4029548,0.08224355,0)^{T}, \quad d Z^{\star} / d t=(-0.05135043,0.1739387,0)^{T} .
$$

Numbers from 0 to 7 show positions of $P$ for $p=0, \frac{1}{4} \pi, \ldots, \frac{7}{4} \pi$ with the difference $\frac{1}{4} \pi$. The sets of points, around them correspond to positions at these times of perturbed motion with initial perturbations $(\delta x(0)=-0.008, \delta z(0)=0.04)$. 

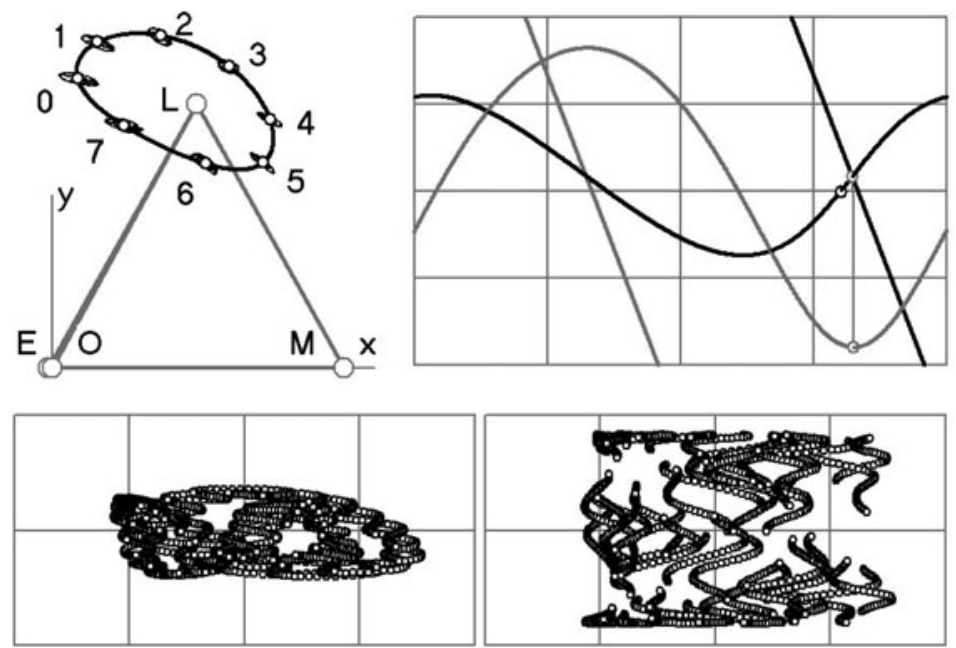

The dependence of angular distance $q=\arcsin \frac{-\sqrt{ } 3(x+\mu)+y}{2 r}$ from $L_{4}$ to $P$ (black curve), and the difference $(r-1)$ (gray curve) on $p$ are shown in the rectangle $(0<p<2 \pi ;-0.2<$ $q, r<0.2)$ at top-right figure. Straight lines show the dependence $p+q=\frac{5}{3} \pi$ (black) for full illumination and $p+q=\frac{2}{3} \pi$ (gray) for absence of illumination of the particle by the Sun. We see that three positions: passage $P$ before $L_{4}$, shortest distance $r=0.82$ of $P$ from the Earth and full illumination of $P$ - are very close to each other. The first corresponds to $t^{\star}=t_{0}+T-\Delta t$ ( $\Delta t$ before full moon), $\Delta t=5^{d} 20^{h} 24^{m}$. The angular velocity of the dust-cloud with respect to stars at this moment equals to $17^{\circ} 57^{\prime}$ per day.

From symmetry of the equations of motion $y \rightarrow-y, p \rightarrow-p$, we get the periodic solution, capturing the point $L_{5}$. Similarly the optimal time to observe the dust-cloud in this case is the same $\Delta t$ after full moon. In some approximation the equations are also invariant for $p \rightarrow p+\pi$. Hence, we get another two $2 \pi$-periodic solutions with the same graph but for $-\pi<p<\pi$ and with the role of the illumination lines interchanged. For both solutions full illumination corresponds to the furthest distance of point $P$ from the Earth $(r=1.164)$.

The numerical experiment shows that the angular radius of the dust cloud is between $6^{\circ}$ and $9^{\circ}$ depending on the direction in the space. The set of positions of the mentioned above perturbed motion at the moments $t^{\star}+k T, k=0, \ldots, 2000$ in rectangles $(0<p<2 \pi,-0.05<r<0.05)$ and $(0<p<2 \pi,-0.05<\arcsin (z / r)<0.05))$ is shown at bottom-left and bottom-right figures. The latter projection corresponds to the view from the Earth. For large perturbations of the initial conditions the motion become chaotic, and the dust density beyond the neighborhood of the periodic orbit decrease.

\section{Implications}

We show the possibility of existence of four dust-clouds. The optimal time for observation of two of them are at the time $\Delta t=5^{d} 20^{h} 24^{m}$ before the full moon in vicinity of $L_{4}$, and at the same time after the full moon in vicinity of $L_{5}$, if it is night and the points $L_{4}$ and $L_{5}$ are up the horizon. Two other clouds are less suitable for observation from the Earth. These theoretical results agree well with the Kordylewski observations and give possibility to predict the optimal time for observations.

\section{Reference}

Kordylewski, K., 1961, Acta Astron., 11, 165 